\title{
Prévenir les gangs de rue en se basant sur la sociologie des organisations : pour sortir des sentiers battus
}

Sylvie Hamel, Martine Vézina et Marie-Marthe Cousineau

\section{(2) OpenEdition Journals}

Édition électronique

URL : http://journals.openedition.org/communicationorganisation/669

DOI : 10.4000/communicationorganisation.669

ISSN : 1775-3546

Éditeur

Presses universitaires de Bordeaux

Édition imprimée

Date de publication : 1 décembre 2008

Pagination : 242-262

ISBN : 978-2-86781-582-9

ISSN : $1168-5549$

\section{Référence électronique}

Sylvie Hamel, Martine Vézina et Marie-Marthe Cousineau, « Prévenir les gangs de rue en se basant sur la sociologie des organisations : pour sortir des sentiers battus ", Communication et organisation [En ligne], 34 | 2008, mis en ligne le 01 décembre 2011, consulté le 10 décembre 2020. URL : http:// journals.openedition.org/communicationorganisation/669; DOI : https://doi.org/10.4000/ communicationorganisation.669 


\section{Expériences}

\section{Résumé}

Cet article fait état d'une expérience vécue à Montréal au Québec, reliée à la prévention du phénomène des gangs de rue. Ce projet, intitulé Jeunesse et gangs de rue, ne recourt pas au moyen traditionnellement utilisé, en l'occurrence la répression, mais s'appuie plutôt sur une approche de développement social communautaire. Pour suivre et analyser cette démarche, les chercheures se basent sur un modèle de la sociologie des organisations. Deux critères sont empruntés à ce modèle, servant à évaluer la qualité du projet qui se développe, soit la solidité de l'objet technique et l'ancrage de la solution dans son milieu. Ce cadre d'analyse pose un regard neuf sur la prévention des gangs de rue en s'intéressant à des dimensions très différentes de celles que la criminologie et la psychologie privilégient. Il indique que Jeunesse et gangs de rue pourrait constituer une innovation sociale.

\section{Mots clés}

Gangs de rue, prévention, développement social, innovation sociale, sociologie des organisations

\section{Summary}

This article states an experience lived in Montreal (Quebec), related to the prevention of street gangs phenomenon. This project, called Jeunesse et gangs de rue (Youth and street gangs), does not appeal to the method traditionally used, in occurrence the repression, but relies instead on the community social development. To follow and analyse this approach, the researchers base themselves on a model of the sociology of organizations. Two standards are taken from this model, serving to evaluate the quality of the project's development process, the solidity of the technical object and the anchorage of the solution in its environment. This analysis framework brings a new glance on street gangs prevention by taking an interest in very different dimensions that criminology and psychology favour. It indicates that Jeunesse et gangs de rue (Youth and street gangs) could constitute a social innovation.

\section{Key-Words}

Street gangs, prevention, social development, social innovation, sociology of organizations

Sylvie Hamel, Ph.D., est Professeure au Département de psychoéducation, Université du Québec à Trois-Rivières.

Martine Vézina, M.Sc., est Professionnelle de recherche à l'Institut de recherche pour le développement social des jeunes à Montréal (Québec)

Marie-Marthe Cousineau, Ph.D., est Professeure à l'École de criminologie, Université de Montréal (Québec). 
Prévenir les gangs de rue...

\title{
Prévenir les gangs de rue en se basant sur la sociologie des organisations : pour sortir des sentiers battus
}

Sylvie Hamel, Martine Vézina, Marie-Marthe Cousineau

\author{
sylvie.hamel@uqtr.ca \\ sylvie.cormier@cjm-iu.qc.ca \\ marie-marthe.cousineau@umontreal.ca
}

Le projet Jeunesse et gangs de rue a été et continue d'être une entreprise de développement social communautaire vouée à la prévention du phénomène des gangs de rue dans la région montréalaise au Québec. Le projet initial fut réalisé entre 2000 et 2005 grâce à un financement provenant des ministères de la Justice et du Solliciteur général du Canada, dans le cadre de la Stratégie nationale pour la sécurité communautaire et la prévention du crime. Dans trois agglomérations des intervenants et des citoyens ont uni leurs compétences pour mettre en œuvre des plans d'action locaux novateurs, visant une augmentation de la participation et de l'intégration sociale des jeunes, misant sur le fait que les efforts ainsi consentis pourraient, en retour, avoir des retombées sur la délinquance des gangs de rue ${ }^{189}$.

\section{État de la situation des gangs de rue}

L'approche de prévention du phénomène des gangs dont nous faisons la promotion dans le cadre du projet Jeunesse et gangs de rue fut d'abord influencée par les premières recherches que nous avons menées sur le sujet des gangs de rue. Ainsi, on a pu constater qu'il y a bien longtemps déjà que le phénomène des gangs de rue existe en Amérique, mais que tout porte à croire qu'il est en pleine progression, entraînant un durcissement de la délinquance de ces groupes (Cousineau, Hamel \& Desmarais, 2004, Venkatesh \& Levitt, 2000). Le Service de police de Montréal évalue que, depuis 1989, sept homicides par année seraient

189 Le projet initial est maintenant terminé, sa conclusion se traduisant par la réalisation d'un guide d'action intersectorielle pour la prévention du phénomène des gangs rédigé par Hamel, S., Cousineau, M-M. et Vézina, M. (2006). Depuis ce temps, les localités participantes poursuivent toujours leurs actions. 


\section{Expériences}

attribuables aux gangs. Depuis 2002, 397 tentatives de meurtre ont également été reliées aux gangs (Barbeau, 2005).

Toutefois, on a du mal à s'entendre sur une définition des gangs. Le terme englobe en fait une diversité de groupes qui ne s'adonnent pas tous à la violence et à la criminalité avec la même intensité (Hébert, Hamel \& Savoie, 1997). Au premier pôle du continuum se situent les groupes de jeunes qui tendent à imiter les gangs tandis qu'à l'autre extrémité se placent les organisations criminelles résolument tournées vers la violence et la criminalité. Entre ces deux limites, se trouvent une quantité de groupes, dont les gangs de rue. Ceux-ci renvoient à la notion de gangstérisme, évoquant la présence d'un phénomène organisé et criminalisé, qui entraîne, du même coup, une réaction sociale fortement antagoniste. De plus, en questionnant de jeunes membres sur des dimensions psychologiques de l'expérience qu'ils ont vécue dans ces groupes, nous en sommes venues à la conclusion qu'au-delà de la violence et de la criminalité qu'il génère, le phénomène des gangs est lié à un autre problème tout aussi important: celui voulant que des jeunes découvrent dans les gangs le moyen de combler leurs besoins les plus fondamentaux (protection, appartenance, reconnaissance et valorisation), besoins qu'ils ne trouvent à combler nulle part ailleurs dans leur environnement (famille, école et communauté) (Hamel, Fredette, Blais \& Bertot. 1998).

\section{Nouvelle lecture de la solution}

Dans cette foulée, nous avons développé l'idée que la prévention des gangs n'est possible qu'en mettant en place les conditions nécessaires pour que « les jeunes s'attachent et s'intègrent à leur communauté comme ils s'attachent et s'intègrent aux gangs ». De cette manière, nous prenions en compte les recommandations d'experts, œuvrant depuis longtemps dans le milieu de criminologie, qui accordent une grande valeur à une approche préventive et communautaire (Spergel, 1995 ; Howell, 1998). Ces derniers sont d'avis que la complexité du phénomène des gangs exige une action renforcée par la combinaison de plusieurs stratégies (mobilisation des collectivités, développement organisationnel, intervention sociale et répression) déployées dans une perspective globale, faisant que les jeunes ne sont pas les seuls visés, mais aussi la famille, l'école et la communauté. 
Il faut dire que les moyens traditionnellement utilisés pour faire face au problème des gangs n'ont eu que peu de succès. En effet, les moyens employés en Amérique pour enrayer la progression des gangs ont consisté, dès le début des années 1980, presque exclusivement en des procédés répressifs (Howell, 2000). Les principaux acteurs de cette guerre aux gangs étaient les différents représentants de l'ordre et de la loi. Ces stratégies ont aussi été employées au Québec, à Montréal particulièrement, depuis le milieu des années 1980 jusqu'au milieu des années 1990. Mais celles-ci n'ont donné lieu à aucun résultat probant (Hébert, Hamel \& Savoie, 1997). C'est pourquoi nous avons choisi de privilégier une autre approche, beaucoup moins répandue cependant : le « développement social communautaire ». Cette stratégie n'est pas centrée sur les situations et les faits criminels, mais sur les causes et sur l'amélioration du tissu social comme moyen de freiner la propagation de gangs et de la délinquance qu'ils génèrent.

Le développement social dépend en grande partie des variables économique et culturelle, avec lesquelles il doit être en étroite relation pour se déployer (Hancock, 2001). Mais encore davantage, le développement social s'appuie sur la population elle-même. Non seulement il contribue à son épanouissement, mais il le fait par l'activation de son propre potentiel. Ceci rejoint la position de Chavis (2000) qui définit le développement social comme un processus de coopération volontaire, d'entraide et de construction de liens sociaux entre les résidents et les institutions d'un milieu local, visant l'amélioration des conditions de vie tant individuelles que collectives, sur les plans physique, social et économique. En somme, nous visions à faire alliance avec des communautés locales dans l'intérêt de les rendre plus attrayantes et plus signifiantes afin que les jeunes y reconnaissent les opportunités qu'elles leur offrent de satisfaire leurs besoins d'apprendre, de se construire et de changer les choses. Dans un tel environnement, la délinquance aurait tendance à perdre de sa vitalité (Hawkins \& Catalano, 1992).

\section{Nouvelle façon de construire la solution}

Nous avons donc choisi de nous allier aux membres de trois quartiers. À titre de localités pilotes, ces quartiers ont été retenus en fonction de deux critères : d'abord parce qu'on les savait préoccupés, de différentes façons, par le phénomène des gangs et aussi parce qu'ils étaient reconnus pour leur expérience plus ou moins réussie de concertation. Dans ces 


\section{Expériences}

agglomérations, ce sont des intervenants que nous avons approchés en premier lieu, formant des comités dont les acteurs provenaient des milieux public, parapublic et communautaire, et pratiquant dans divers secteurs d'intervention interpellés par la cause des jeunes, tels l'éducation, l'emploi et la solidarité sociale, la justice, les affaires municipales et la métropole, les relations avec les citoyens et l'immigration, la santé et les services sociaux, ainsi que la sécurité publique. À ces intervenants se sont alliés aussi des jeunes et des parents, citoyens soucieux du bien-être de la jeunesse.

\section{Nouvelle lecture du rôle de la recherche}

Au commencement du projet, il fallut évidemment présenter la vision que nous avions du problème des gangs et des solutions à y apporter. Mais l'équipe de recherche a du également donner des précisions sur la façon dont elle comptait se mettre en lien avec les milieux. Nous voulions nous y intégrer afin de participer aux changements souhaités. Notre proposition: la réalisation d'une recherche participative. Le premier argument à l' appui de la recherche participative repose sur le fait que nous voulions construire avec les milieux le programme d'action découlant de la théorie que nous avancions, plutôt que de l'imposer et de l'expérimenter. Nous envisagions même que cette théorie puisse être bonifiée par les acteurs en tentant de la rendre opérationnelle. Comme pour toutes recherches participatives, la théorie sur laquelle nous nous appuyions n'était pas vouée à la prédiction (Wadsworth, 1998) mais à la validation et à l'appropriation des connaissances.

Il nous fallait donc recourir aux communautés elles-mêmes, à leurs expertises, à leur connaissance des jeunes, à leurs moyens et à leurs ressources. Ceci rejoint l'idée de plus en plus répandue selon laquelle les preuves scientifiques ne sont pas suffisantes à la conception des programmes et, en conséquence, que la participation des milieux s'impose comme dimension incontournable et essentielle à l'élaboration de l'action des pouvoirs publics. La complexité des problèmes auxquels nous sommes aujourd'hui confrontés, ici le phénomène des gangs, requiert plutôt que des mécanismes soient mis en place permettant qu'une diversité d'acteurs concernés négocient, s'ajustent les uns aux autres selon leur pouvoir relatif et leurs systèmes de valeurs respectifs, pour prendre ensemble des décisions issues d'ententes et de compromis. En fait, nous ne croyons pas que les programmes naissent et se déploient autour d'une 
logique exclusivement scientifique, qui renvoie aux principes d'exhaustivité et de cohérence. Les programmes les plus prometteurs s'inscrivent aussi dans une logique d'intérêt, d'effets d'entraînement et de compromis qui s'appuie elle-même sur des principes de légitimité et de crédibilité (Bilodeau, 2000).

\section{Deux visions du transfert de la connaissance}

Deux grandes visions se dessinent autour du transfert de connaissance. Dans l'une, on conçoit que celui-ci s'appuie sur la diffusion des résultats de la recherche ou encore sur des stratégies de changement planifié. Le processus du transfert de la connaissance est dans ce cas représenté de manière linéaire. Cette vision propose une séquence rationnelle, commençant d'abord par la recherche, suivie de la formatisation du savoir, de la diffusion de masse, de la planification et la division des tâches, si une action doit en découler. Cependant, il a été maintes fois constaté qu'il ne suffit pas de diffuser la connaissance pour que celle-ci soit utilisée (Yin \& Gwaltney, 1981).

Dans l'autre vision, qui est celle à laquelle nous adhérons, le processus du transfert de la connaissance est conçu de manière plus complexe. Il est vu comme s'insérant dans une relation dynamique entre le chercheur et l'utilisateur de la connaissance. En s'appropriant la connaissance, ce dernier contribue à la transformer pour qu'ainsi elle puisse mieux se véhiculer et voyager dans des réseaux sur lesquels il exerce une influence (Gélinas, 1988). Dans cette perspective, le chercheur s'associe étroitement à l'utilisateur dès le début de sa recherche, parce qu'il le considère comme porteur d'une vision valide du problème auquel il s'intéresse, y étant lié par l'action. En agissant, l'humain est pragmatique, engagé et cherche naturellement des moyens pour résoudre les problèmes qui se posent à lui. C'est pourquoi, le chercheur ne prescrit pas la solution mais facilite son émergence chez l'utilisateur. En revanche, le chercheur possède un avantage que l'agissant n'a pas. Son approche rationnelle lui permet d'élever le savoir tacite et d'expérience de l'utilisateur à un degré supérieur, celui d'une pratique consciente faisant partie intégrante de la connaissance à transférer. Celle-ci fait alors l'équilibre entre la théorie et la pratique (Bouchard \& Gélinas, 1989).

Cette vision du transfert de la connaissance se situe très près de celle que nous retrouvons dans la théorie sociologique de la traduction, proposée par Michel Callon (1986). Nous résumons ici cette théorie qui encadre les 


\section{Expériences}

analyses qui ont été faites tout au long de notre projet. Les résultats qui en ressortent, comme nous le verrons plus loin, mettent en évidence des dimensions qui, à notre avis, ouvrent sur de nouvelles pistes de réflexion à propos de la prévention du phénomène des gangs par le développement social communautaire.

\section{La théorie sociologique de la traduction}

Dans le langage courant, une traduction constitue une «forme d'activité de compréhension consistant dans le passage d'une communication d'une forme de langage à une autre » (Office québécois de la langue française, 2007). Pour Michel Callon et Bruno Latour (1986), ce qui relève de la traduction s'inscrit tout à fait dans le cadre de cette définition générale, à ceci près que le passage d'une langue à une autre est remplacé par la recomposition d'une information, d'un message, d'un objet. Traduire c'est aussi déplacer et opérer une translation, c'est-à-dire un mouvement. Suivre une innovation, c'est suivre la succession des interprétations et réinterprétations de ses composantes. En d'autres mots, les gens qui s'emparent d'une innovation le font selon leurs intérêts et souvent la transforment finalement en quelque chose d'autre. Si les nombreux intérêts de divers groupes d'acteurs sont alignés, l'innovation va se répandre comme une traînée de poudre ; s'ils ne le sont pas, l'innovation n'aura pas lieu. Ou bien l'objet se transforme, en intéresse d'autres et donc se déplace et dure ; ou bien il est inachevé, n'intéresse personne et s'éteint. Dans ce modèle, l'objet ne se trans-porte que s'il se trans-forme, contrairement à ce que supposent les modèles traditionnels et linéaires de la diffusion de la connaissance. La traduction évolue donc au fil de l'agencement séquentiel des trames technique et sociale de l'évolution d'un projet. La trajectoire qu'elle emprunte en est une tourbillonnaire et non linéaire. Il s'agit dès lors d'examiner le jeu des allers et retours, des interactions entre les éléments du technogramme (trame technique) et ceux du sociogramme (trame sociale). L'innovation est le fruit des adaptations, des ajustements, des articulations entre ces entités.

Ainsi, pour établir la qualité d'une innovation sociale, deux dimensions sont à considérer. D'une part, la solidité de l'objet technique est un premier élément fondamental. Sa qualité repose en partie sur ses fondements, mais également sur sa vraisemblance et sur sa pertinence qui lui assurent une viabilité. Un objet de qualité est celui qui d'abord est bien confectionné, mais qui également trouve de véritables preneurs, c'est-à- 
dire qu'il intéresse des acteurs qui y voient un potentiel, estiment en fait que l'objet est utile et adéquat. Le deuxième critère servant à établir la qualité d'une innovation sociale tient à l'ancrage de la solution dans son milieu. C'est-à-dire que, dans un deuxième temps, un objet technique de qualité est censé conduire les premiers acteurs qu'il a su intéresser à mobiliser d'autres acteurs de leur communauté qui, à leur tour, le trouveront également nécessaire. À cette étape, le réseau doit cependant affronter et résoudre ses controverses et se rallier autour de points de convergence. C'est ainsi qu'il pourra se consolider et continuer de transporter l'innovation sociale. En d'autres mots, la solidité de l'innovation dépend de l'irréversibilité du réseau qui le porte. Ce mécanisme fait en sorte que le réseau se fortifie en multipliant les unités qui le composent et en positionnant celles-ci stratégiquement à la manière dont s'enroulent les granules de sucre autour du bâtonnet dans la confection de la barbe à papa. Bien entendu, ceci n'est possible que dans la mesure où l'objet est adapté et subit un certain nombre de transformations en cours de route qui, pour leur part, facilitent les déplacements de l'objet au sein du réseau. Les résultats obtenus en usant de ces critères, soit la solidité de l'objet technique et l'ancrage de la solution dans son milieu, pour analyser le déroulement des travaux de Jeunesse et gangs de rue s'avèrent fort intéressants. Pour illustration, nous présentons ici une unique figure de cas, soit celle d'un des quartiers pilotes (que nous appelons localité A), s'agissant d'un des premiers à Montréal à avoir connu l'émergence des gangs dans la forme qu'on leur connaît aujourd'hui. Cette illustration est suffisante en soi pour enrichir nos réflexions sur le développement social communautaire comme moyen de prévenir ce problème.

\section{Le cas de la localité A}

Rappelons que le but de la recherche évaluative et participative que nous avons menée dans le cadre du projet Jeunesse et gangs de rue était de documenter la construction, la mise en œuvre, de même que les retombées de la stratégie de prévention par le développement social communautaire.

\section{Éléments méthodologiques}

L'étude de cas est la stratégie méthodologique que nous avons employée (Grawitz 1993). L'analyse, qui est inductive, possède aussi une visée explicative (grounded theory) puisqu'elle cherche non seulement à déterminer quelles sont les conditions favorisant la réussite des 


\section{Expériences}

changements de pratiques d'intervention en matière de gangs de rue, mais s'efforce aussi de comprendre comment ces conditions agissent (liens fonctionnels) sur cette réussite. Cet examen s'exécute de manière itérative en développant progressivement l'explication à partir d'une série d'opérations interreliées auxquelles se greffent progressivement de nouvelles propositions théoriques de plus en plus précises, jusqu'à l'obtention d'un modèle théorique d'évaluation ajusté à la réalité observée (Yin, 1989). Le tableau qui suit regroupe les principales composantes de la théorie du problème et de l'action dont nous avons fait la promotion dans le cadre de ce projet.

\begin{tabular}{|c|c|c|}
\hline Théorie du problème & \multicolumn{2}{|l|}{ Théorie de l'action } \\
\hline Position & Philosophie & $\begin{array}{l}\text { Principes directeurs de } \\
\text { l'action }\end{array}$ \\
\hline \multirow{4}{*}{$\begin{array}{l}\text { 1. Les jeunes } \\
\text { découvrent dans les gangs } \\
\text { le moyen de combler leurs } \\
\text { besoins les plus } \\
\text { fondamentaux (protection, } \\
\text { appartenance, } \\
\text { reconnaissance } \\
\text { valorisation), moyen } \\
\text { qu'ils ne trouvent nulle } \\
\text { part ailleurs dans leur } \\
\text { environnement (famille, } \\
\text { école et communauté). }\end{array}$} & \multirow{4}{*}{$\begin{array}{l}\text { 2. La prévention } \\
\text { des gangs tient à la mise } \\
\text { en place des conditions } \\
\text { nécessaires pour que les } \\
\text { jeunes s'attachent et } \\
\text { s'intègrent à leur } \\
\text { communauté comme ils } \\
\text { s'attachent et s'intègrent } \\
\text { aux gangs. }\end{array}$} & $\begin{array}{l}\text { 3. perspective } \\
\text { multistratégique } \\
\text { (sensibilisation, } \\
\text { opportunités, } \\
\text { intervention, } \\
\text { mobilisation, } \\
\text { répression; sur un } \\
\text { continuum } \\
\text { d'intervention); }\end{array}$ \\
\hline & & $\begin{array}{l}4 . \quad \text { approche } \\
\text { globale ciblant jeunes, } \\
\text { écoles, } \quad \text { familles, } \\
\text { communauté ; }\end{array}$ \\
\hline & & \begin{tabular}{llll}
$\mathbf{5}$ & \multicolumn{3}{c}{ reconnaissance } \\
des forces & et & de \\
l'autonomie & & des \\
individus & & & et \\
collectivités ; & & &
\end{tabular} \\
\hline & & $\begin{array}{|ll|}\mathbf{6 .} & \text { développement } \\
\text { social. } & \end{array}$ \\
\hline
\end{tabular}

Composantes du modèle théorique sur le phénomène des gangs à l'essai : problème et action

Les données recueillies pour procéder à cette analyse, toutes qualitatives, proviennent de sources variées: procès-verbaux des réunions de travail 
des comités d'action locale; entrevues semi-dirigées réalisées chaque année avec des acteurs clés de chacun des groupes ; documents de travail utilisés par les comités; rapports d'activités de l'équipe de recherche; rapports annuels des organismes partenaires; documents d'information, sites Internet sur les partenaires et leurs services; observations participantes. L'ensemble du matériel recueilli fut traité de façon convergente en suivant un mode de triangulation. Il fut codifié à partir d'une grille développée à cette fin, utilisée avec une intention de conceptualisation en tentant de relier les différents éléments de données, tout en les confrontant aux éléments du modèle théorique de départ. Tout au long du processus d'analyse, la corroboration - qui se base essentiellement sur l'échange d'interprétations entre les membres de l'équipe de recherche (Poupart, Deslauriers, Groulx, Laperrière, Mayer \& Pires, 1997) - fut privilégiée pour le contrôle de la validité interne.

Diverses informations ont également été amassées pour tracer le portrait des localités qui allaient être investiguées. Ces portraits ont d'abord été constitués à partir de données officielles sur les caractéristiques sociodémographiques et économiques des milieux, ainsi que différents indicateurs se rapportant de plus près à la criminalité. Dans le cas qui nous occupe ici, celui de la localité A, dix-sept intervenants œuvrant dans la localité, ont été joints au tout début du projet. Ces derniers ont répondu à un sondage téléphonique où ils étaient conviés à faire état de ce qu'ils savaient de l'étendue et des manifestations du phénomène des gangs dans leur localité et des actions qu'ils souhaitaient voir se réaliser pour y faire face. Enfin, une enquête fut conduite dans chacune des localités pour sonder les résidents de 10 à 65 ans de même que les commerçants sur leurs perceptions à l'égard du climat social régnant dans la localité. À cette fin, les entrevues réalisées s'articulaient autour d'un ensemble de thèmes couverts dans un questionnaire écrit voulant mesurer diverses dimensions de la vie de quartier.

\section{Résultats}

\section{Le portrait abrégé de la localité A}

Les intervenants ayant répondu au sondage téléphonique ont clairement indiqué qu'ils avaient une préoccupation pour les gangs. Ils estiment que ces groupes sont bien présents dans le quartier et ceci depuis un bon moment déjà. Ces gangs s'adonnent à diverses activités telles la vente de stupéfiants, le proxénétisme, les vols qualifiés, les voies de fait et 


\section{Expériences}

l'intimidation. Les rassemblements de ces jeunes sont perçus comme dérangeants et engendrent un sentiment d'insécurité au sein de la population, que les médias entretiennent, dans une certaine mesure, par l'entremise de la couverture souvent sensationnaliste qu'ils font des événements ayant lieu sur le territoire.

Les données de l'enquête sur le climat social viennent à leur tour corroborer que la localité A présente une problématique de gangs de rue importante. En effet, 12,3\% de l'ensemble des répondants questionnés dans ce quartier estiment que les gangs de rue sont très présents au sein de leur localité. Devant ce portrait qui suscite l'inquiétude face au phénomène des gangs dans la localité $\mathrm{A}$, les intervenants se préoccupent aussi des moyens à prendre pour y faire face. À cet égard, leurs propos sont nuancés, l'expérience leur ayant démontré qu'une intervention massive et centrée sur la coercition ne donne pas les effets escomptés. Les intervenants appuient plutôt l'idée que, pour faire face à ce problème, l'intervention doit être portée par les acteurs de tous les organismes et de toutes les organisations afin de créer les conditions nécessaires à l'intégration sociale des jeunes.

\section{La solidité de l'objet technique}

La localité A a solidement adhéré au modèle théorique proposé au départ. Les principes se trouvant à la base de ce modèle ont été transportés et même bonifiés dans un imposant cadre de référence, prenant la forme d'un document substantiel d'une vingtaine de pages. Il reprend les éléments théoriques du modèle de départ, auxquels s'ajoutent des éléments pratiques.

\section{Résumé de la philosophie d'intervention du comité d'action locale de la localité A}

\section{Principales caractéristiques de notre approche d'intervention}

- Nos actions doivent cibler tant les jeunes que leur environnement. C'est toutefois aux interventions auprès des jeunes que nous accordons la priorité. Quant aux interventions auprès de l'environnement, c'est toujours en fonction des jeunes que nous entendons les faire.

- Afin d'éviter de morceler l'intervention, différents acteurs doivent être mis à contribution (famille, école, milieu de travail, communauté, etc.). La Table de concertation jeunesse favorise ici la concertation intersectorielle afin de faire le pont entre ces différents acteurs. 
- Nous préconisons une approche multistratégique (accès aux structures traditionnelles, développement de structures alternatives, intervention sociale, répression, etc.). Les stratégies mises de l'avant devront être intégrées dans un continuum d'intervention.

- Il importe de miser d'abord sur les forces des jeunes eux-mêmes, qu'ils soient ou non membres de gangs. Pour ce faire, nous devons faire appel aux ressources de chaque jeune et de ses pairs. Cette approche n'écarte pas la possibilité de solliciter un appui extérieur.

- Nos actions doivent s'adresser à tous les jeunes afin d'éviter le cloisonnement entre différents groupes de jeunes. L'exclusion est considérée comme une mesure temporaire lorsqu'elle s'avère nécessaire.

- L'articulation des interventions à mener doit se faire à travers les activités régulières des organismes ou des projets spécifiques mettant à contribution un ou plusieurs organismes.

Synthèse des pistes d'action proposées

But : Prévenir le développement du phénomène des gangs de rue dans la communauté et ce, tout en étant solidaire des autres communautés.

\section{Pistes d'action s'adressant aux jeunes en général et à leur} environnement

\section{Jeunes}

- Mettre en place des projets leur permettant de se développer pleinement, de s'exprimer, d'innover, de se réunir dans des groupes à orientation pro-sociale, de s'engager activement dans la vie communautaire et sociale et de se donner un projet de vie stimulant incluant des valeurs spirituelles.

- Mettre les jeunes en présence d'adultes significatifs avec qui ils peuvent établir des liens positifs. Leur offrir des modèles positifs.

- Sensibiliser les jeunes à l'importance de s'associer à des pairs positifs et à résister aux pairs négatifs. Mettre l'accent sur les conséquences associées à la violence et à la délinquance plutôt que sur la fréquentation des gangs de rue.

- Développer des activités de loisirs où le plaisir défait des barrières et permet que des liens se tissent.

\section{Famille}

- Créer un environnement favorisant l'épanouissement des différentes personnes membres de la famille. 


\section{École}

- Rendre l'école plus attrayante tant au plan scolaire que parascolaire. Favoriser le travail concerté avec le milieu.

- Rendre l'école plus sécuritaire et régler, autant que possible, les problèmes à l'interne.

Travail

- Contribuer au développement d'emplois intéressants (conditions de travail, milieux de travail).

- Rendre les milieux de travail habiles à soutenir leurs employés.

Loisirs

- Développer des loisirs répondant mieux aux besoins des jeunes.

- Rendre intéressants et soutenant les milieux de loisirs.

- Rendre disponible ou créer des lieux répondants aux besoins des jeunes (sport, culture, musique, etc.).

\section{Communauté, quartier, rue}

- Améliorer la perception que les résidents ont des jeunes en général

- Faire en sorte que la rue, les parcs, etc., deviennent des lieux sécuritaires pour les jeunes et la population en général.

- Lutter contre la pauvreté.

- Favoriser le rapprochement interculturel.

- Créer un tissu social plus dense.

- Favoriser l'accès à des lieux de rassemblement.

\section{Société}

- Si nécessaire, interpeller les décideurs (politique jeunesse, politiques de lutte contre la pauvreté, politique interculturelle, politique familiale, politique éducative, politique d'emploi, politique des loisirs, etc.).

Pistes d'actions s'adressant spécifiquement aux jeunes plus vulnérables face au phénomène des gangs et à leur environnement

\section{Jeunes}

- Leur offrir un accueil et des possibilités d'obtenir un soutien adéquat.

- Leur offrir des activités qui répondent positivement aux besoins auxquels répondent les gangs et pour lesquels ils y sont attirés (appartenance, pouvoir, sécurité). 
Prévenir les gangs de rue...

\section{Pistes d'actions s'adressant spécifiquement aux jeunes associés aux gangs de rue et à leur environnement}

\section{Jeunes}

- Réprimer les comportements de ceux qui se trouvent à la tête de la délinquance et le faire dans une démarche d'aide.

- Offrir des occasions de remettre en question leurs choix de faire appel à des comportements délinquants et de faire partie de ces gangs et ce, tout en leur proposant des solutions de rechange susceptibles de les intéresser.

\section{Famille}

- Interpeller les parents afin de leur apprendre que leur enfant fait partie de l'univers des gangs et solliciter leur collaboration dans la réhabilitation de leur enfant.

- Soutenir les familles en difficulté.

Par contre, vingt réunions du comité d'action, s'étalant sur 36 mois de travail ont été consacrées à la conception de ce cadre de référence. Le temps dédié à la réflexion dans cette localité peut sembler exagéré de prime abord. Toutefois, cet exercice était essentiel avant de pouvoir passer à l'action. En effet, plusieurs enjeux ont d'abord dû être dénoués avant de pouvoir procéder. Des interventions passées avaient causé de nombreuses tensions au sein de la concertation locale, et en conséquence la communauté avait cessé, depuis de nombreuses années, de chercher des solutions au problème des gangs de rue. L'intervention auprès de ces groupes avait alors été placée sous l'entière responsabilité des policiers. Avec l'arrivée du projet qui remettait le sujet des gangs de rue à l'ordre du jour, il est devenu indispensable que ces points de tension soient examinés à nouveau. Dans ce contexte, la problématisation (le positionnement des acteurs face au problème) s'avérait une étape cruciale, bien que difficile. Avant de parvenir au développement d'une vision commune du phénomène des gangs et des solutions à mettre de l'avant pour y faire face, les acteurs de cette localité se sont frottés à de nombreuses controverses qu'ils se sont affairés à résoudre à mesure qu'elles émergeaient au fil des discussions. Parmi celles-ci nous comptons, par exemple, les débats ayant eu lieu à propos de la concertation et du temps qui doit lui être accordée. Les intervenants dont le travail exige qu'ils soient constamment mobilisés pour des urgences s'accommodaient difficilement du temps qui doit être réservé à la réflexion. Certains étaient d'avis que l'énergie consacrée à la concertation aurait été mieux 


\section{Expériences}

rentabilisée si elle avait été employée à «s'attaquer» directement aux gangs. Mais ne trouvant aucun appui au sein du comité, ces derniers se sont finalement retirés. En somme, le projet Jeunesse et gangs de rue a conduit les membres de la localité A à entreprendre un virage paradigmatique important, faisant en sorte que le comité d'action s'est vu ébranlé d'abord par une crise identitaire considérable. Dans un milieu habitué à réagir contre les gangs plutôt que d'y faire face par d'autres moyens, le processus d'appropriation du nouveau modèle proposé au départ a conduit les membres du comité à renégocier leur position et leur pouvoir respectif sur l'échiquier de la concertation. C'est pourquoi une entente claire devait être établie entre eux, apparaissant dans un cadre de référence affirmant l'adhésion du groupe au modèle de départ avec un certain nombre d'alinéas permettant d'intégrer de manière consensuelle le plus grand nombre de positions possibles.

Au terme de cette étape, le comité, qui durant les premiers mois du projet avait reçu de nombreux visiteurs et connu d'importants changements au plan de sa composition, était formé à part égale de représentants des milieux communautaires et institutionnels. Ces derniers étaient principalement issus des secteurs de la santé et des services sociaux, avec quelques délégués des milieux de l'éducation et de la sécurité publique. Ensemble, ils se ralliaient au cadre de référence qu'ils avaient développé et souhaitaient maintenant définir les opérations qui allaient rendre possible sa mise en œuvre.

\section{L'ancrage de la solution dans son milieu}

En mai 2002, ce comité déposait à la Table de concertation jeunesse le bilan de ses actions et ses perspectives pour l'année à venir. Il annonçait alors qu'il voulait faire la promotion du cadre de référence en tant que tel afin que l'ensemble de la communauté puisse en bénéficier. Aucune autre instance ne pouvait aussi bien faire ce travail, dont la charge était beaucoup plus réaliste pour le comité que de se river à l'idée de mettre en œuvre les actions proposées, avec des moyens réduits. En faisant la promotion du cadre de référence, il se donnait de meilleures chances de s'allier à d'autres forces qui, en se joignant à lui, allaient l'aider à porter les actions que le cadre proposait. Avec cette crédibilité nouvelle que leur avait procuré la production d'un cadre de référence, les membres du comité furent invités à le présenter à d'autres instances actives et influentes dans la municipalité. Chacune de ces rencontres ont permis au 
comité de s'enraciner davantage dans la communauté et de préciser les projets et les activités qu'il voulait réaliser. Ces liens se sont formalisés au point où ces autres entités sont devenues de nouveaux partenaires officiels.

En mai 2003, le plan d'action du comité d'acteurs s'articulait autour de trois axes principaux. Le premier se consacrait toujours à la promotion du cadre de référence; le deuxième, aux perceptions des membres de la communauté, voulant par là rétablir les faits qui ont déjà terni son image ; et le troisième, se vouait à la prévention par la mise en place et la consolidation de projets répondant aux besoins des jeunes. Un an plus tard, le réseau continuait de s'agrandir. La reconnaissance et la visibilité acquises au fil des ans permettaient au comité d'action de solliciter divers partenaires dont la contribution s'avérait dorénavant essentielle à l'avancement des travaux. Il s'agissait d'acteurs clés, dont les policiers et le milieu scolaire, ayant présenté de fortes résistances au début du projet mais qui, finalement, allaient céder devant la force d'entraînement du réseau. Le comité a également pu interpeller l'administration municipale qui, dans le passé, ne s'était pas jointe aux actions de ses membres. Cette fois, elle les appuyait de manière formelle, ainsi que leurs nouveaux alliés, en leur donnant un financement stable permettant d'implanter un important projet au sein du quartier: l'Animation du milieu urbain qui, encore aujourd'hui, se déploie dans la localité et ne cesse d'interpeller de nouveaux partenaires. Ce projet veut répondre au fait que de plus en plus de jeunes du quartier fréquentent désormais la rue, les parcs et autres endroits publics, n'ayant nulle part ailleurs où aller pour socialiser et se divertir. Pour éviter que la situation ne se détériore davantage, et parce que celle-ci est propice à la formation de gangs, il est apparu capital pour la communauté que des intervenants animent ces lieux, y assurent une présence, recréent d'une certaine façon ce que les jeunes peuvent retrouver dans les maisons de jeunes (activités sportives, culturelles et ludiques), mais à ciel ouvert. Pour son fonctionnement, le projet Animation du milieu urbain s'appuie sur la mobilisation de l'ensemble des ressources du milieu pour aller vers les jeunes, là où ils se trouvent, et pour qu'ils disposent gratuitement d'un maximum de lieux et d'activités en dehors des heures de classe.

En 2004, le projet mettait en scène les intervenants de trois maisons de jeunes, du service de police, de la municipalité, de deux écoles secondaires, de deux centres pour jeunes raccrocheurs et de deux 


\section{Expériences}

entreprises d'économie sociale qui, ensemble, ont réussi à joindre 7922 jeunes qui ont participé à des activités régulières et ponctuelles (événements spéciaux). En 2005, 9631 jeunes se sont inscrits à des activités régulières et 1404 , à des activités ponctuelles (Hamel, Cousineau \& Vézina, 2006). En 2006, le projet était mis en candidature pour recevoir le prix d'excellence du Réseau québécois de Villes et Villages en santé qui se consacre aux projets de développement durable. Ceci indique que le projet initial est resté fidèle aux principes de son modèle de départ et que le réseau qui le porte se consolide et s'allonge constamment. À cela s'ajoute également le fait que les porteurs du projet de l'Animation du milieu urbain sont aujourd'hui responsables du développement d'un cursus de formation provinciale destinée à tous les acteurs pouvant être appelés à faire face au phénomène des gangs.

\section{Conclusion}

La démarche que nous venons d'illustrer offre un certain contraste vis-àvis des stratégies habituelles visant à contrer le phénomène des gangs. Il faut dire que de nombreuses recherches ont documenté son ampleur, les activités délinquantes et les manifestations violentes qui s'y rattachent, ce qui fut de nature à soulever dans les communautés la volonté d'éradiquer les gangs plutôt que de mobiliser des ressources pour s'occuper des jeunes qui en font partie. En conséquence, de nombreuses stratégies de prévention situationnelle ont été déployées afin de faire face à ce problème. Mais, ce type de prévention qui a pour fonction de protéger des personnes et des biens contre des menaces précises n'a que peu d'emprise sur le phénomène des gangs. Les experts recommandent plutôt une approche globale, à multiples dimensions, s'articulant sur une base locale. En d'autres mots, il s'agit de faire du développement social communautaire une stratégie ambitieuse qui elle aussi renferme un certain nombre de pièges.

À cet égard, Cusson (2002), tout en concédant que le développement social veut s'attaquer aux causes profondes de la criminalité, notamment à la misère des gens qui développent ce style de vie comme moyen de subsistance, déplore que le plein emploi, qui pourrait être conçu comme $l a$ solution, soit un objectif à toutes fins utiles impossible à réaliser. Hastings (1998), adoptant une vision moins restrictive, soutient de son côté que le développement social communautaire peut favoriser l'autonomisation des communautés locales par une mobilisation des ressources servant à la 
mise sur pied de programmes réalistes et efficaces. Mais Hasting indique que cette stratégie, comme toutes les autres, présente aussi ses limites, dont celles du localisme. L'auteur dénonce le fait que les milieux peuvent s'épuiser à lutter contre les causes d'un problème lorsqu'elles dépassent les frontières de leur communauté.

Quoi qu'il en soit, il semble que la mobilisation dans la localité A soit bien réelle et celle-ci connaît une montée qui aujourd'hui commence à dépasser les frontières locales de cette communauté. À vrai dire, nous croyons que cette initiative devrait être considérée avec la plus grande attention. Avec le regard neuf qui permet de poser sur elle la théorie sociologique de la traduction, cette expérience apparaît être des plus prometteuses. Elle nous en enseigne en premier lieu que la prévention du phénomène des gangs est une entreprise fort complexe, ne s'agissant pas seulement de faire face à la criminalité que génèrent ces groupes, mais de considérer également la jeunesse des personnes qui en font partie. Cette dualité peut soulever un certain nombre de tensions, notamment celles entre deux perspectives passablement différentes, le contrôle et le développement social. Ceci rend certaines opérations délicates et peut prolonger le temps nécessaire au développement de liens de confiance sur lesquels doit s'appuyer les partenaires pour pouvoir faire face à ce problème. En conséquence, les acteurs de ce milieu ont développé un solide cadre de référence, de même qu'un plan d'action dont l'un des objectifs est centré sur la cohérence, l'échange et la communication. Dans cette foulée, nous constatons que les membres de cette localité sont parvenus à créer un véritable effet d'entraînement dans leur communauté. Avec le temps, le réseau s'est considérablement élargi, joignant à chaque fois de nouveaux effectifs et de nouvelles ressources servant à l'enrichissement de son plan d'action.

Puis c'est de manière graduelle que les membres du groupe d'action se sont rapprochés du phénomène des gangs, sans perdre de vue que leur mission première consiste à s'occuper des jeunes qui en font partie. Après s'être occupés du réseau d'action, les cibles qu'ils ont visées sont les jeunes en général, puis les jeunes à risque tout en incluant les jeunes victimes. En toute logique, le réseau sera bientôt assez solide pour s'occuper des jeunes membres de gangs, soit de ceux qui présentent le plus haut niveau de difficulté au plan de la cohérence des opérations. La situation de ces jeunes ravive en effet la possibilité d'une polarisation des points de vue, en mettant nez à nez deux enjeux très différents, celui de la 


\section{Expériences}

sécurité publique d'une part et celui de la protection et du développement de l'enfance d'autre part. En d'autres mots, ce milieu a résisté à d'importants enjeux moraux qui auraient pu le conduire à procéder de manière différente, voulant qu'il s'occupe en premier lieu et en toute urgence des jeunes qui sont le plus à risque ou encore de ceux qui vivent de graves problèmes avec les gangs. Mais il a préféré éviter, en connaissance de cause, les conséquences qu'auraient avoir sur ces jeunes des actions incohérentes et mal gérées entreprises par un réseau d'acteurs qui ne se font pas confiance. Un tel réseau ne saurait sécuriser ces jeunes et leur donner envie de s'y joindre plutôt que de se lier aux gangs.

\section{Bibliographie}

BARDEAU, Danielle, Actualité Gang De Rue: Bilan des projets du SPVM 2005. Publication du Service de police de la Ville de Montréal, 2005, 6 p.

BILODEAU, Angèle, «Les conditions de qualité de la planification participative et de l'action en partenariat dans le champ de la prévention promotion de la santé et du bien-être, le cas des priorités régionales 19951998 de Montréal-Centre », Thèse de doctorat, Université de Montréal, 2000.

BOUCHARD, Y. \& GÉLINAS, A. «Un modèle alternatif de formation de futurs chercheurs », in Revue de l'ARQ, 1990, vol. 3, pp. 119-141.

CALLON, Michel, «Éléments pour une sociologie de la traduction: la domestication des coquilles Saint-Jacques et des marins-pêcheurs dans la baie de Saint-Brieuc» in L'Année sociologique, 1986, vol. 36, pp. 169-208.

CALLON, Michel \& LATOUR, Bruno, (1986), «Les paradoxes de la modernité : comment concevoir les innovations?» in Prospective et santé, vol. 36, pp. 13-25.

CHAVIS, David. M., «Mobilizing Community Capacity Building», Présentation au Laboratoire de recherche en écologie humaine et sociale, Université du Québec à Montréal, 28 avril 2000.

COUSINEAU, Marie-Marthe et al., "Faits saillants de la consultation provinciale sur les jeunes et le phénomène des gangs au Québec », dans les Actes du Forum provincial: Pour la création d'un réseau québécois permanent d'échanges sur les jeunes et les gangs de rue : les gangs de rue 
Prévenir les gangs de rue...

faut plus qu'en parler, Solliciteur général du Canada et Ministère de la sécurité publique du Québec, 2004, pp. 31-36.

CUSSON, Maurice, Prévenir la délinquance, les méthodes efficaces. Paris : Presses universitaires de France, 2002, 200 p.

GÉLINAS, A., Essai sur la nature et les modalités du programme des subventions pour études et analyses. Rapport de recherche commanditée, Conseil québécois de la recherche sociale, 1988.

GRAWITZ, Madeleine, Méthodes des sciences sociales. Paris : Dalloz, 1993, $920 \mathrm{p}$.

HAMEL, Sylvie, et al., "Jeunesse et gangs de rue : évaluation d'un projet d'action intersectorielle pour la prévention du phénomène des gangs ». Institut de recherche pour le développement social des jeunes, pour le Centre national pour la prévention du crime, 2006, $256 \mathrm{p}$.

HAMEL, Sylvie, et al., « Jeunesse et gangs de rue (phase II) : résultats de la recherche-terrain et proposition d'un plan stratégique quinquennal», Rapport soumis au Service de police de la Communauté urbaine de Montréal, Montréal : Institut de recherche pour le développement social des jeunes, 1998, $440 \mathrm{p}$.

HANCOCK, Trevor, Health-based Indicators of Sustainable Human Development. Paper prepared for the Office of Sustainable Development, Health Canada, 2001.

HASTINGS, Ross, «La prévention du crime par le développement social : une stratégie à la recherche d'une synthèse », dans Criminologie, 1998, vol. 31, pp. 109-123.

HAWKINS, David. J. \& CATALANO, Richard F., Communities that care: Action for Drug Abuse Prevention. San Francisco: Jossey-Bass Publishers, 1992.

HÉBERT, Jacques et al., « Jeunesse et gangs de rue (phase I) : revue de littérature ». Rapport soumis au Service de police de la Communauté urbaine de Montréal. Montréal: Institut de recherche pour le développement social des jeunes, 1997, 100 p.

HOWELL, James C., Youth Gangs: An Overview, Washington : Office of Juvenile Justice and Delinquency Prevention ( OJJDP ), 1998.

HOWELL, James C., Youth Gang Programs and Strategies. Washington: Department of Justice, Office of Juvenile Justice Programs, Office of Juvenile Justice and Delinquency Prevention, 2000. 


\section{Expériences}

Office québécois de la langue française, 2007, http://www.oqlf.gouv.qc.ca/ 8 février 2007.

POUPART, Jean, et al., La recherche qualitative: enjeux épistémologiques et méthodologiques. Montréal : Gaëtan Morin, 1997, $405 \mathrm{p}$.

SPERGEL, Irwin A., The Youth Gang Problem: A Community Approach. New York: Oxford University Press, 1995, 346 p.

VENKATESH, Sudhir A. \& LEVITT, Steven D. «Are we a Family or a Business? History and Disjuncture in the Urban American Street Gang », in Theory and Society, $\mathrm{n}^{\circ}$ 4, août 2000, pp. 427-462.

WADSWORTH, Yoland, "What is participatory action research ?" Action Research International. Paper 2, 1998.

YIN, Robert K., \& GWALTNEY, Margaret.K., « Knowledge utilization as a networking process » in Knowledge : Creation, Diffusion, Utilization. 1981, vol. 2, pp. 555-580.

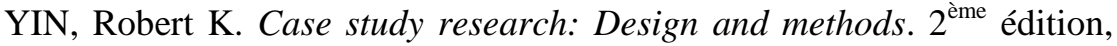
Newbury Park : Sage Publications, 1989, 131 p. 